\title{
س
}

$>\mathrm{DE}$

$\stackrel{1=1}{\simeq}$ PÉDAGOGIE

\author{
Recherches en éducation
}

180 | juillet-août-septembre 2012

Le CAP : Regards croisés sur un diplôme centenaire

\section{Penser autrement les carrières scolaires}

Thinking school paths differently

Pensar diferentemente las carreras escolares

Die Schulkarriere umdenken

Philippe Masson

\section{OpenEdition \\ Journals}

\section{Édition électronique}

URL : https://journals.openedition.org/rfp/3821

DOI : 10.4000/rfp.3821

ISSN : 2105-2913

Éditeur

ENS Éditions

Édition imprimée

Date de publication : 15 juillet 2012

Pagination : 121-130

ISBN : 978-2-84788-388-6

ISSN : 0556-7807

\section{Référence électronique}

Philippe Masson, "Penser autrement les carrières scolaires », Revue française de pédagogie [En ligne], 180 | juillet-août-septembre 2012, mis en ligne le 15 juillet 2012, consulté le 21 septembre 2021. URL http://journals.openedition.org/rfp/3821 ; DOI : https://doi.org/10.4000/rfp.3821 


\section{POSITIONS, DÉBATS ET CONTROVERSES}

\section{Penser autrement les carrières scolaires}

À la suite d'un numéro récent de la Revue française de pédagogie (n० 175), l'auteur examine d'autres orientations possibles pour l'étude des " choix scolaires ». II souligne les conséquences pratiques qui découlent de l'usage de cette terminologie et propose de lui substituer la notion de "carrière scolaire » qui permet d'élargir la recherche à d'autres situations de choix scolaires souvent peu étudiées. II présente un cadre d'analyse pour l'étude des carrières scolaires et les pistes de recherche qui pourraient en découler.

Mots-clés (TESE) : orientation, parcours scolaire, choix d'une école, choix de la formation, attitude envers l'école, scolarisation.

Faut-il repenser les choix scolaires? La Revue française de pédagogie vient de consacrer un de ses derniers numéros ( ${ }^{\circ}{ }^{175}$ ) à cette question ${ }^{1}$. Le contexte politique et institutionnel de l'Éducation nationale, en particulier avec la réforme de la carte scolaire, a favorisé les recherches sur les choix de filières de scolarisation, d'options, ou d'établissements. Au-delà du contexte dans lequel elles s'inscrivent, quelques questions paraissent importantes dans ce dossier : comment les choix scolaires sont-ils influencés par les ressources des individus ? La capacité à choisir est-elle socialement distribuée ? Peut-on penser les trajectoires individuelles sur le mode de l'alternance entre des formes de déterminisme et de rationalisme ? Et quelles relations entre ces choix et les inégalités sociales? Ces questions sont associées à des enjeux conceptuels (autour de la notion de choix), à des enjeux méthodologiques (comment les étudier ?) et à des "enjeux portant sur le choix de la population à étudier » (Blanchard \& Cayouette-
Remblière, 2011, p. 6). Je voudrais ici contribuer à ce débat, en proposant une approche qui apparaîtra probablement décalée par rapport à ces questions, et plus largement, par rapport aux orientations et préoccupations actuelles de la sociologie française de l'éducation. Mon intention est de suggérer une autre manière d'appréhender les problèmes, apte à renouveler nos questions et nos terrains de recherche.

\section{LE TERME DE CHOIX RESTREINT NOTRE MANIÈRE D'ENVISAGER LES PROBLÈMES}

Dans la sociologie de l'éducation française, il est d'usage d'opposer, au moins depuis la fin des années soixante, le déterminisme des conditions objectives des trajectoires scolaires au rationalisme des choix, aux stratégies subjectives que prétendrait saisir 
l'individualisme méthodologique. Cette opposition est présentée depuis longtemps comme un élément structurant la spécialité 2 . Elle s'est progressivement imposée dans les manuels de la spécialité en France, devenant ainsi une "question scolastique » (Bourdieu, 1997) à laquelle les sociologues semblent devoir sacrifier avant de proposer leurs propres analyses, parce qu'elle paraît fondamentale alors qu'elle relève de la métaphysique et ne peut être tranchée. D'ailleurs, la question ne date-t-elle pas un peu ? Sans verser dans le subjectivisme d'une sociologie de l'expérience ou celui d'une "sociologie de l'individu ", reconnaissons que les travaux sur la philosophie de l'action, qu'ils soient anglo-saxons - comme ceux d'Élisabeth Anscombe (2002) ou ceux, à l'opposé, de Donald Davidson (1993), s'appuyant sur les sciences cognitives - ou français - tel Vincent Descombes (2004) -, ont conduit à repenser les problèmes. Ce n'est pas le lieu pour en discuter. Soulignons juste que faire un choix scolaire est une action intentionnelle. Ce n'est pas une simple action volontaire (comme lever le bras, pour reprendre un exemple de Wittgenstein), ou une question abstraite, mais au contraire une pratique très concrète : laquelle des trois cases de ce dossier d'orientation vais-je cocher : seconde générale, seconde professionnelle ou redoublement ? II y a une réelle intentionnalité de l'action parce que les parents ou les élèves peuvent donner différents récits de la manière dont est prise la décision. Nous autres, sociologues, le savons bien. En pratique, c'est bien parce que les gens peuvent donner un récit et une justification de leurs actions que nous les interviewons. Que l'on se contente de leurs paroles ou que l'on y trouve, au contraire, des indices du fait qu'ils n'ont pas complètement conscience de ce qu'ils font, de ce qui les pousse à agir, est un autre problème.

Les parents et les élèves délibèrent pour résoudre un problème pratique, car c'est d'abord ainsi que se présente le choix scolaire. Pour en rendre compte, nombre de sociologues continuent d'opposer deux grands types de déterminations. Les uns mettront l'accent sur la cohérence de toutes les pratiques d'une personne en renvoyant celle-ci à sa socialisation primaire, à ses dispositions incorporées. II s'agira alors de mettre au jour l'ensemble des conditions qui déterminent l'incorporation de ces dispositions, et, par là, des comportements semblables chez tous ceux qui occupent une même position dans la société. Les autres insisteront sur le caractère indéterminé et créatif des interactions. Ils chercheront à dégager l'ensemble des éléments de contextualisation qui permettent de rendre compte de la manière dont les gens définissent leur situation et agissent en conséquence. Ce dilemme, qui semble tarauder les socio- logues, n'existe pas en fait. II n'y a aucune difficulté logique à accepter l'existence de ces deux types de déterminations. La littérature sociologique présente des exemples, parfois anciens, de la combinaison en acte, dans la recherche empirique, de ces deux types de déterminations ${ }^{3}$. Ainsi, Jean-Michel Chapoulie (1987, p. 130) montre que les rapports des professeurs au fonctionnement de l'enseignement secondaire au milieu des années soixante-dix dépendent à la fois de " leurs intérêts matériels ou symboliques déterminés par la position sociale occupée dans l'enseignement et hors de l'enseignement » et de leur expérience sociale du recrutement. Celle-ci est saisie aussi bien dans ses aspects objectifs, c'est-à-dire les différentes étapes de la carrière scolaire et professionnelle, que dans ses " aspects subjectifs, les significations vécues dont a été investie cette trajectoire ". Une analyse comparable pourrait être effectuée à propos du vote électoral, autre domaine dans lequel chacun est appelé à faire un choix. Seule l'enquête empirique permet d'établir en pratique le domaine de pertinence de ces deux types de déterminations et la manière dont ils s'appliquent.

\section{Question politique, question morale}

Marianne Blanchard et Joanie Cayouette-Remblière (2011, p. 6) soulignent, avec raison, que les sociologues paraissent mal à l'aise avec le terme de choix. Ils jugent utile de le mettre entre guillemets, comme le fait Choukri Ben Ayed dans son article, " afin de ne pas naturaliser la question ", dit-il $(2011$, p. 56$)$, ou espérant ainsi éviter de trancher le dilemme purement théorique et scolastique que j'évoquais plus haut. Le terme de choix appliqué à l'orientation scolaire (ou à celui de l'établissement) peut poser une question politique relative au traitement des inégalités sociales à l'école. Une politique de lutte contre ces inégalités peut être différente selon que l'on considère que les familles ont le libre choix de leur orientation, et font un choix en conscience, ou que leurs choix ne sont que le résultat inconscient de contraintes objectives qu'il faudrait parvenir à corriger. C'est cette question qui est implicitement au centre du contrepoint de Stéphane Beaud dans ce même dossier (Beaud, 2011). La politique de "libéralisation » de l'école - pour reprendre ses termes - accorde une place importante, au moins dans les discours officiels, à cette notion de choix (en particulier dans la réforme de la carte scolaire). Aussi Stéphane Beaud juge-t-il utile de rappeler qu'au-delà des choix scolaires s'imposent d'abord des rapports de classes. Mais une question morale se profile aussi derrière cette question politique. Les sociologues, et les spécialistes de l'éducation en particulier, jugent 
plus moralement acceptable de défendre les classes populaires que de justifier sociologiquement la mise en œuvre de politiques qui semblent les desservir. La question n'est pas de savoir si nous pourrions faire, ou non, une sociologie sans valeurs. C'est, là aussi, un dilemme imaginaire. La question est plutôt de savoir " de quel côté nous sommes » (Becker, 1967).

Choukri Ben Ayed remarque, au début de son article, que les recherches sociologiques sur les choix scolaires sont depuis longtemps étroitement liées au contexte politique de l'Éducation nationale. Au début des années quatre-vingt, des sociologues s'interrogeaient sur les effets des premières mesures d'assouplissement de la carte scolaire. Ils interprétaient les choix scolaires des parents comme des effets de comportements stratégiques. Les politiques récentes de désectorisation ont relancé les travaux sur les choix scolaires. Comme on pouvait s'y attendre, les travaux ont d'abord porté sur les classes moyennes et supérieures, probablement directement visées par ces politiques. Les questions politiques apparaissent ainsi comme source de recherches sociologiques. Les sociologues peuvent ainsi souhaiter évaluer la politique de libéralisation de l'offre scolaire mise en œuvre par le ministère de l'Éducation nationale. Si l'on suit Sylvain Broccolichi et Rémi Sinthon (2011), ce sont encore des questions politiques et institutionnelles qui semblent intervenir dans l'interprétation qu'Alain Girard et Alfred Sauvy (1965) faisaient des tables de scolarité du suivi longitudinal de l'Institut national d'études démographiques dans les années soixante. Broccolichi et Sinthon notent ainsi que "nous entrevoyons à quel point la production des données, le filtrage des informations et l'orientation des analyses peuvent se trouver affectés par des enjeux politico-institutionnels ou corporatistes : soutenir les politiques menées, préserver des légitimités institutionnelles en rapport notamment avec le mérite scolaire [...], conforter la pertinence de certains courants de recherche " (2011, p. 30). Suffit-il, pour élargir le champ d'étude, de s'intéresser aux élèves des classes populaires ? C'est ici qu'apparaissent les "enjeux méthodologiques" (comment étudier les choix ?) et les "enjeux portant sur le choix de la population à étudier » (Blanchard \& Cayouette-Remblière, 2011, p. 6). Autrement dit, il s'agit d'une question d'échantillonnage.

\section{Question d'échantillonnage, question sociologique}

Les travaux qui ont porté ces dernières années sur les choix scolaires ont pris pour objet le choix d'établissement (autour des questions relatives à la carte scolaire), les choix de filières et d'options, et plus largement les processus d'orientation au sein des établissements. Ils ont donc porté sur des questions relatives à la scolarisation, et pas tant sur l'éducation ${ }^{4}$. Les niveaux d'enseignement choisis pour analyser ces choix scolaires furent surtout le collège et le lycée, et dans une moindre mesure, une partie de l'enseignement supérieur (l'université et les sections de techniciens supérieurs). Ces recherches sur les choix scolaires se sont principalement intéressées aux classes moyennes et supérieures (Van Zanten, 2009 ; Gombert \& Van Zanten, 2004 ; Oberti, 2007). II semble clair que ces classes sociales ont, dans ce cas, un rapport positif à l'institution scolaire. D'autres travaux récents ont montré que les familles de classes populaires acceptaient d'autant plus aisément l'orientation de leurs enfants vers des études longues que leurs notes scolaires étaient satisfaisantes. À l'opposé, quand les verdicts scolaires sont négatifs, ils s'excluent beaucoup plus que les classes moyennes ou supérieures de ce type de cursus. Certains y voient les nouveaux visages de l'auto-exclusion populaire (Poullaouec, 2010). Le rapport à l'école aurait donc changé au fur et à mesure de sa généralisation. Dans les années soixante-dix, les sociologues britanniques avaient, au contraire, souligné les comportements de résistance des familles populaires à l'égard de l'école, qui pouvaient impliquer un rapport de méfiance, voire de défiance, vis-à-vis de l'institution. Ce n'est pas tant la pertinence ou non de ces analyses qui m'intéresse ici que le fait qu'elles envisagent toutes la question du choix des familles à l'intérieur de l'institution scolaire. La sociologie de l'éducation a toujours été implicitement une sociologie de l'éducation scolaire. Le comportement dans et à l'égard de l'école est la préoccupation centrale des sociologues de l'éducation, même si, paradoxalement, la transmission des savoirs - un des aspects importants de l'école au regard des finalités qu'elle s'attribue - n'est pas la chose la plus étudiée.

On sait pourtant qu'avant de choisir l'établissement, il faut d'abord vouloir s'inscrire, opter pour la fréquentation scolaire. Certes, celle-ci est aujourd'hui largement acquise, dès le plus jeune âge, puisque $95 \%$ des enfants de 4 ans sont scolarisés à l'école maternelle. Les nomades, ou gens du voyage, peuvent faire des choix entre se fixer sur un terrain ou une zone géographique restreinte, voire se sédentariser, pour permettre la scolarisation de leurs enfants, ou continuer à voyager, comme le souligne Daniel Bizeul (1993, p. 96-97) dans son enquête ethnographique ${ }^{5}$. Des familles en situation irrégulière sur le territoire peuvent être contraintes de retirer leurs enfants de 
Tableau 1. Le rapport à la scolarisation

\begin{tabular}{|l|l|l|}
\cline { 2 - 3 } \multicolumn{1}{c|}{} & \multicolumn{1}{c|}{ Avant ou après l'école } & \multicolumn{1}{c|}{ Dans l'école } \\
\hline $\begin{array}{l}\text { Rapport } \\
\text { positif }\end{array}$ & choix de la fréquentation scolaire & $\begin{array}{l}\text { choix de l'établissement, d'une filière, d'une } \\
\text { option } \\
\text { nouveau visage de l'auto-exclusion }\end{array}$ \\
\hline $\begin{array}{l}\text { Rapport } \\
\text { négatif }\end{array}$ & $\begin{array}{l}\text { non-inscription ou fréquentation irrégulière } \\
\text { choix de l'emploi } \\
\text { choix de l'apprentissage }\end{array}$ & résistance \\
\hline
\end{tabular}

l'école dans le cas d'une menace d'expulsion, ou au contraire les y inscrire, en espérant éviter celle-ci. Le choix n'est donc pas uniquement l'apanage des classes sociales aisées, bien diplômées, et disposant d'une bonne connaissance des rouages de l'institution. Des parents bien diplômés peuvent décider de ne pas scolariser leurs enfants dans des établissements scolaires et choisir de leur faire eux-mêmes la classe (en complétant, parfois, par une inscription au CNED). Bien sûr, ces cas restent marginaux, mais ils existent. C'est en prenant en compte les cas «à la marge " que nous pouvons élargir le champ du concept de choix. Le tableau 1 présente quelques exemples possibles des attitudes face à la scolarisation et à l'institution scolaire.

Certes, certains des comportements évoqués sont minoritaires face à la masse des familles qui utilisent régulièrement l'institution scolaire. La question n'est pas, en fait, celle du nombre, mais de l'attention aux catégories sociales les moins visibles, les moins légitimes de l'espace social. Aussi pourrions-nous nous intéresser à la gamme complète des variations d'un phénomène, plutôt qu'à une partie de celui-ci, fûtelle centrale. Ainsi, nous éviterions de " confondre un cas spécifique avec toute la classe de phénomènes à laquelle il appartient » (Becker, 2002, p. 229). En n'omettant pas une seule des possibilités pour lesquelles une famille doit se prononcer à l'égard de l'école, à commencer par celle de la fréquentation scolaire, nous élargissons le champ du concept et découvrons alors que la question du choix est incluse dans un processus plus large.

\section{LES CARRIÈRES SCOLAIRES COMME PROCESSUS ET COMME INTERACTION}

La fréquentation scolaire et ses modalités à tous les niveaux de l'enseignement, le choix entre le public et le privé (quand l'offre scolaire le permet), le type d'établissement, le parcours au sein de celui-ci, la durée de la scolarisation, l'usage qui est fait de la scolarisation et du diplôme sont autant d'aspects d'une carrière scolaire. Le terme de carrière a l'avantage d'englober l'ensemble des étapes au cours desquelles les élèves et leurs familles sont conduits à se prononcer, à faire des choix, en matière de scolarisation. II permet aussi de ne pas nous limiter aux dimensions politique et morale, évoquées plus haut, qu'induit le concept de choix. Cette carrière doit être envisagée comme un processus. Elle se présente comme une suite de paliers où à chaque étape des bifurcations se présentent, les familles et les élèves peuvent choisir telle ou telle possibilité. C'est sur le long terme que se construit le rapport à l'école, un investissement ou non dans l'institution scolaire. Envisager la carrière scolaire comme un processus revient alors à prendre pour objet « la dialectique entre ce qui est régulier et récurrent d'un côté, et ce qui est unique de l'autre ; une telle étude [...] vise ainsi à se placer au point de rencontre entre une société stable mais néanmoins changeante, et l'être humain unique » (Hughes, 1996, p. 176-177). Les familles et les élèves ne sont évidemment pas les seuls à prendre des décisions qui peuvent influer sur les carrières scolaires. L'ouverture ou non d'une option, la fermeture d'une classe, la volonté d'agir sur le processus d'orientation des élèves selon des normes statistiques, par exemple, sont des possibilités offertes au chef d'établissement qui contribuent à la définition de l'offre scolaire (Masson, 1999). J'ai ainsi souligné, comme le rappellent Marianne Blanchard et Joanie Cayouette-Remblière (2011), que la scolarité d'un élève est aussi influencée par les interactions entre toutes les catégories de personnes qui participent à la vie des établissements.

Deux points essentiels découlent de cette perspective : d'une part, les carrières scolaires ne peuvent pas être examinées en dehors du cadre géographique dans lequel elles s'insèrent ; d'autre part, parce qu'il 
s'agit d'un processus, donc changeant, et que les carrières se déroulent toujours dans une zone géographique donnée, il n'y a pas de comportement unifié à une classe sociale nominalement définie. Ces deux points méritent quelques explications. Les chercheurs en sciences sociales ont toujours observé des variations géographiques dans la pratique religieuse (et le rapport à la religion), même quand celle-ci était largement dominante en France. De ce point de vue, la Vendée n'est pas le Var. De même, quand les politistes et les historiens examinent les résultats électoraux, ou plus largement le rapport au politique, ils soulignent les variations géographiques de ces pratiques. Là non plus, la Vendée n'est pas le Var. Des historiens, comme Maurice Agulhon, Paul Bois ou Alain Corbin, ont souligné, chacun à leur manière, les variations géographiques des pratiques politiques, du rapport au politique au sein d'un département ou d'une région. Dans le domaine de la santé, l'utilisation de l'offre de soins médicaux varie aussi d'une région à l'autre, tout comme varie la situation sanitaire des populations. Bref, il semble banal de rappeler que le rapport aux institutions varie d'une zone géographique à l'autre. Les sociologues de l'éducation semblent, depuis près d'une vingtaine d'années, porter davantage d'intérêt à la " dimension locale " des faits scolaires, en particulier quand ils travaillent sur les inégalités territoriales de réussite scolaire (par exemple, Broccolichi, Ben Ayed \& Trancart, 2006, 2010). Ils cherchent généralement à dégager des " effets de contexte ", le local (une commune, une zone urbaine, un département, etc.) devenant ainsi une des variables d'ajustement au même titre que «l'effet-maître » ou « l'effet-établissement ». II ne s'agit pas seulement de dire que dans telle zone urbaine, telle classe sociale ne respecte pas la carte scolaire en utilisant le choix des options proposées par les établissements secondaires, ou encore que la réussite scolaire est meilleure dans tel département que dans tel autre mais, que le rapport de chaque classe sociale à l'école n'est pas homogène sur l'ensemble du territoire et, plus fondamentalement, que les interactions entre une population socialement diversifiée et une institution sont géographiquement définies.

Sur le long terme, la situation des classes sociales change, les contours de chaque classe sociale évoluent, tout comme leur contenu. Les ouvriers d'hier ne sont pas ceux d'aujourd'hui. Plus encore, pour une période donnée, Jean Peneff (1987) a bien montré que, dans une commune, il n'y avait pas sur la scolarisation de comportement unifié à une classe sociale nominalement définie, parce que le comportement de chacune est le fruit des interactions qu'elles ont entre elles.
Ainsi, à Soullans (en Vendée), il montre que « quand les fils des indigents ou des domestiques étaient les premiers à occuper les bancs de la "communale", les petits ou les moyens agriculteurs mirent du temps à se convaincre qu'ils y avaient leur place. Quand la masse des paysans alla à l'école, l' "élite" de la commune trouva déplaisant de s'y associer et préféra se retirer, choisissant d'autres modes de scolarisation (à domicile, en pension). Quand les familles républicaines soutinrent l'école communale, les familles catholiques se montrèrent aussitôt réservées ou inversement. Ce furent souvent les interactions de groupes qui déterminèrent les comportements et non pas une caractéristique immuable propre à telle catégorie " (Peneff, 1987, p. 144). De plus, l'offre de formation n'est pas géographiquement répartie de façon homogène, aussi bien en ce qui concerne la distinction entre le public et le privé, que pour les différents niveaux d'enseignement. À terme, cette offre s'étoffe et se modifie et le rapport d'une classe sociale à l'école évolue parfois rapidement, comme le rappelle Tristan Poullaouec (2011) dans son contrepoint en évoquant les monographies de Laurence Wylie qui analyse l'évolution des attitudes paysannes et ouvrières à l'égard de l'école dans deux environnements très différents (le département de Maine-et-Loire et celui du Vaucluse). C'est au regard de l'offre scolaire existante dans leur environnement que les familles font leurs choix.

Dès lors, nous pourrions faire varier l'échantillon de nos méthodes pour étudier les choix scolaires des familles. Les premiers travaux qui, dans les années quatre-vingt, s'intéressaient aux choix des parents dans le cadre des mesures d'assouplissement de la carte scolaire s'appuyaient sur des enquêtes par questionnaires. Par la suite, les recherches plus récentes sur l'étude des choix scolaires ont reposé encore sur des enquêtes par questionnaires, sur l'analyse secondaire de données - à partir des panels de l'Éducation nationale - et sur des entretiens auprès des familles. On ne saurait trop rappeler que les méthodes ne sont pas que de simples techniques, qu'elles engagent des orientations théoriques, même si certaines méthodes peuvent s'accorder avec plusieurs cadres théoriques. Mais, surtout, elles induisent des manières de concevoir les problèmes.

\section{DE LA NÉCESSITÉ DES MONOGRAPHIES}

Le cadre que je viens de définir permet d'envisager de nouvelles orientations dans l'analyse des carrières scolaires. Le développement de l'approche ethnogra- 
phique en sociologie de l'éducation depuis la fin des années quatre-vingt nous a permis d'avoir une meilleure connaissance du fonctionnement de l'institution scolaire, au moins dans certaines de ses composantes. Mais si l'enseignement secondaire général et technologique, l'enseignement primaire et, dans une moindre mesure, l'enseignement universitaire sont bien connus, nous ne disposons pas assez de monographies sur le fonctionnement des établissements qui relèvent des deux extrêmes de la hiérarchie scolaire : les maisons familiales rurales, l'apprentissage $^{6}$, l'enseignement professionnel, d'un côté, les écoles des élites de la République, de l'autre. À ceux qui pensent que la sociologie de l'éducation ne vaut que si elle est une anthropologie du pouvoir, alors appelons de nos vœux une monographie, par exemple par observation participante, sur le fonctionnement de l'École normale supérieure de la rue d'Ulm, de l'Institut d'études politiques de Paris, de l'École des hautes études en sciences sociales ou de l'École nationale supérieure des mines? Ce dont nous ne disposons pas jusqu'à présent. Bref, une ethnographie des écoles du pouvoir qui pourrait partir de l'analyse plus globale du « champ des grandes écoles " (Bourdieu, 1989).

Parce que la sociologie de l'éducation a été, jusqu'à une date récente, une sociologie dans et de l'institution scolaire, nous pourrions reprendre à notre compte la phrase d'Alain Corbin à propos de l'histoire sociale du $\mathrm{XIX}$ siècle : " nous ne [savons] rien de ce qu'il serait important de savoir dans la perspective d'une histoire du sujet » (Corbin, 1998, p. 10). Comment se construit sur une vingtaine d'années, au fur et à mesure de la scolarité du premier enfant, le rapport à l'école au sein d'une famille ? Comment se combinent, dans les choix scolaires à faire à chaque étape du cursus, l'expérience scolaire des parents, voire des grandsparents ou d'autres membres de la famille, et les interactions avec les différentes catégories de personnels des établissements scolaires (des écoles primaires jusqu'au lycée), dans le cadre d'une offre scolaire localement définie? Dans quelles circonstances intervient l'influence du groupe des pairs ou du marché local de l'emploi ? Bref, une sociologie des carrières scolaires non pas saisies à partir des seules données produites par l'institution scolaire (les fameux panels de l'Éducation nationale), mais à partir du vécu des familles. Une première possibilité serait de pouvoir suivre sur le long terme, à partir d'entretiens ethnographiques (Beaud, 1996), des familles, dans une zone géographique donnée, à chaque étape du cursus scolaire de leurs enfants ${ }^{7}$. Une variante serait de suivre ou de reconstruire les carrières scolaires des enfants d'une même classe de sixième, comme le réalise actuel- lement Matéo Sorin dans le cadre d'un master de sociologie à l'université de Nantes. Dans une même catégorie sociale, comme celle des agriculteurs ou des ouvriers, le rapport des familles à l'école dépend, entre autres choses, du passé scolaire des parents immédiats ou plus lointains qui rendent envisageable tel type de parcours. Quand on analyse les parcours scolaires de deux familles de petits agriculteurs du même village du Morbihan (Masson \& Suteau, 2010), on peut mettre au jour ce que d'aucuns appelleraient un « capital scolaire générationnel » : une ancienneté de la scolarisation et un rapport au politique différent, qui remontent à plusieurs générations. Dans une des familles, ce « capital » permet de comprendre un plus grand investissement dans l'école publique et de plus longues carrières scolaires, tandis que l'autre choisit l'enseignement privé et délaisse l'école, parfois avant même l'obtention des diplômes. Une autre possibilité serait aussi, comme nous le montrions dans cet article, d'étudier des généalogies scolaires de familles, ce qui permettrait à la fois de saisir les différences internes en leur sein - qui sont plus fréquentes qu'il n'y paraît - et les effets (ou le manque d'effets) de l'école en termes de mobilité professionnelle et sociale selon les générations. Dans tous les cas, on ne pourrait pas se départir d'une attention soutenue aux caractéristiques de l'offre scolaire saisie sur le long terme. Ces monographies familiales nous conduiraient à constater que ce n'est pas un type d'organisation scolaire en lui-même qui détermine son usage par une génération et ses effets sur celle-ci, comme le soulignent Jean-Pierre Briand et Jean-Michel Chapoulie pour l'enseignement primaire supérieur en évoquant, dans une nouvelle préface de leur livre, les entretiens qu'ils ont réalisés auprès d'anciens élèves (Briand \& Chapoulie, 2011, p. VII). Plus largement, les possibilités que je viens d'évoquer pourraient aussi constituer des illustrations en actes de la " demande sociale d'éducation ", si fréquemment évoquée par les sociologues depuis le début des années soixante-dix, sans qu'ils réussissent à donner un contenu empirique à cette notion.

Une autre orientation possible viendrait des monographies qui chercheraient à saisir les comportements scolaires des classes sociales dans leur environnement. Depuis le milieu des années quatre-vingt, les recherches de sociologie de l'éducation qui ont adopté une approche ethnographique se sont multipliées. De nombreux travaux ont mis clairement en évidence la dimension locale des faits scolaires, principalement en étudiant des zones socialement et géographiquement spécifiées (Zones d'éducation prioritaire [ZEP], collèges de banlieue, beaux quartiers, etc.). La dimension locale a été principalement utilisée soit comme 
un moyen pour mieux comprendre le fonctionnement interne de l'institution et de ses établissements au regard de la production des inégalités scolaires - par exemple, en tentant de dégager l'effet-établissement (Cousin, 1998) -, soit comme le cadre dans lequel devaient être analysés certains aspects de la politique scolaire (ZEP, carte scolaire, etc.). La dimension locale n'y est pas pour autant considérée comme centrale comme elle l'est chez les géographes (par exemple, Hérin \& Rouault, 1994), où l'on chercherait ainsi à montrer la disparité de la scolarisation d'une zone géographique à l'autre, ou la variété des pratiques scolaires des classes sociales sur un territoire donné. II ne s'agit pas de territoires administrativement définis (commune, département, région), car l'on sait bien que ces zones administratives ne sont pas homogènes (le haut bocage vendéen, autour des Herbiers, ne ressemble pas à la plaine du sud du département autour de Fontenay-le-Comte, et le rapport à la politique y est différent). Si la dimension locale, géographique, est le contexte de l'enquête sociologique, elle n'est pas considérée comme la variable centrale qui détermine la scolarisation. Elle n'est pas l'objet de la recherche, mais simplement le cadre de celle-ci. Quand les sociologues ou les historiens étudient les comportements envers d'autres institutions (comme la pratique religieuse, le vote, la consommation médicale), ils dévoilent, comme je l'ai rappelé plus haut, des conduites spécifiques à certains territoires. $Y$ a-t-il des territoires scolaires ? Les classes sociales ontelles, pour chacune d'entre elles, des comportements homogènes sur l'ensemble du territoire national ? Ou bien ceux-ci dépendent-ils de l'offre scolaire géographiquement différenciée ? Les interactions entre les classes sociales dans le domaine scolaire sont-elles les mêmes partout, ici et là ? Ne pourrions-nous pas développer une histoire sociale de la scolarisation, même récente, qui, saisie par le bas (Prost, 1993), permettrait d'analyser finement des différences internes au sein de telle ou telle classe sociale ${ }^{8}$ ?

S'éloigne-t-on ici de la question des choix scolaires ? Je ne le pense pas. Les choix des familles s'inscrivent dans un territoire défini - dont les contours sont un des objets de l'enquête - tant par les caractéristiques de son offre scolaire que par les interactions entre les classes sociales qui y vivent. Les rapports de classes ne sont pas au-delà des choix scolaires, sauf à donner un sens très étroit à ce dernier terme. En effet, les interactions entre classes sociales (quelles que soient leurs formes, y compris celle de la lutte) ne s'observent pas uniquement autour des débats sur la carte scolaire ou l'évitement des élèves des cités. Elles prennent aussi forme dans les choix politiques des élus locaux (municipalités, conseils généraux ou conseils régionaux) en matière de scolarisation et de financement des établissements (par exemple, pour l'enseignement privé ou l'enseignement public). Les familles et les élèves ne sont pas les seuls à faire des " choix scolaires". Ce qui ouvre ici de nouvelles perspectives de recherche sur les choix effectués par les collectivités territoriales qui ont largement investi, sur la période récente, dans le développement des collèges, des lycées et de l'enseignement supérieur ${ }^{9}$.

\section{CONCLUSION}

S'il est utile de multiplier les monographies pour mieux comprendre les " choix scolaires », pour mieux analyser les carrières scolaires, ce n'est pas pour satisfaire à un illusoire épuisement du réel (idée qui n'existe dans aucune science), ni pour répondre à une question théorique artificielle, mais en raison du caractère provisoire et partiel du savoir sociologique. Les autres sciences ont passé plusieurs siècles à décrire avant de théoriser. De nouvelles monographies pourraient donc être entreprises, à l'instar de celles évoquées par Tristan Poullaouec pour les années soixante, qui nous permettraient de sortir d'une sociologie de l'éducation qui définit souvent ses questions à partir de celles que se pose l'institution scolaire elle-même. Certes, les monographies locales souffrent encore d'une faible considération en sociologie, et elles sont plutôt réservées aux mémoires de master ou aux thèses. Mais ce sont les monographies qui permettent de complexifier notre savoir et qui remettent en question nos théories. J'ai voulu suggérer que, dans ce domaine, nous en savons finalement peu. Penser autrement les « choix scolaires » nous permettrait d'élargir l'éventail de nos interrogations et de nos recherches en commençant d'abord par élargir le champ de ce concept. Comme le soulignait un sociologue américain célèbre, «prendre en compte ces possibles [...] est ce qui permet à la science sociologique d'avancer, en apprenant de nos erreurs, en ajoutant plus et plus encore à ce que nous savons déjà, même lorsque nous découvrons, de plus en plus, le peu que nous savons » (Becker, 2007).

Philippe Masson

philippe.masson@univ-nantes.fr Université de Nantes, UFR de sociologie 
1 Je remercie Tristan Poullaouec, Cédric Hugrée et Marc Suteau pour leurs remarques sur une version antérieure de ce texte.

2 Les comptes rendus du livre de Raymond Boudon, L'inégalité des chances (1973), par Philippe Bénéton dans la Revue française de science politique en 1975, ainsi que celui de Jean-René Tréanton dans la Revue française de sociologie, la même année, sont organisés autour de l'opposition Bourdieu-Boudon, censée illustrer cette dialectique entre déterminisme et rationalité. Comme si les concepts et la relation dialectique qu'ils induisent pouvaient s'incarner et se réduire, ad nominen, dans des ouvrages.

3 Voir, par exemple, Howard Becker (1970, chap. 11).

4 Sur cette distinction importante dans la sociologie de l'éducation, voir Briand, Chapoulie \& Péretz (1980, p. 23-25). Je laisse de côté, dans cette partie, la question de la transmission des savoirs qui conduirait à considérer de nouvelles dimensions et à élargir la problématique à d'autres institutions que l'institution scolaire puisque celle-ci n'est pas la seule à transmettre des savoirs.

5 II indique que, dans les jeunes couples, " ce souci est d'autant plus énoncé que l'implantation locale [de la famille] remonte à plusieurs décennies, déjà inaugurée par les parents, sous la force fréquemment des nécessités économiques et réglementaires, suscitant dès lors la prise en compte des façons dominantes de vivre (le bénéfice des allocations familiales, essentiel comme source de revenus pour de nombreuses familles, est en particulier conditionné par l'assiduité scolaire) » (Bizeul, 1993, p 97).

6 Parmi les rares travaux récents, qui adoptent une large perspective historique, sur les écoles d'apprentissage, citons ceux d'Emmanue Quenson (2001) pour Renault et de Nicolas Hatzfeld (1989) pour Peugeot.

7 On comprend aisément la rareté de ce type d'enquête, qui s'étalerait sur deux décennies, face aux contraintes productivistes et aux normes de publication qui pèsent sur la carrière des enseignants chercheurs et des chercheurs aujourd'hui. Une autre contrainte est aussi celle des offres de financement sous forme de contrats de recherche. Les orientations de la sociologie de l'éducation dépendent aussi, comme pour d'autres spécialités de la discipline, du contexte de travail des chercheurs et enseignants chercheurs.

8 Dans le même ordre d'idées, bien que les sociologues aient longuement débattu ces dernières années de la démocratisation de l'enseignement au terme de la politique des $80 \%$ au baccalauréat menée à partir de 1985, ils n'ont pas entrepris une évaluation de celle-ci à partir des lycées d'une ville, à l'instar du travail d'Antoine Prost (1992) sur Orléans (réalisé pour la période 1959-1985).

9 Voir Marc Suteau (1999), dont les recherches portent sur une période ancienne (1830-1940) et dont on peut regretter qu'elles n'aient pas servi d'exemple pour l'analyse de la période récente.

\section{BIBLIOGRAPHIE}

ANSCOMBE É. (2002). L'intention. Paris : Gallimard.

BEAUD S. (1996). " L'usage de l'entretien en sciences sociales. Plaidoyer pour l'entretien ethnographique ". Politix, no 35, p. 226-257.

BEAUD S. (2011). "Par delà les choix scolaires : les rapports de classes ". Revue française de pédagogie, no 175 , p. $77-80$.

BECKER H. S. (1967). "Which side are we on? ». Social Problems, vol. 14, p. 239-247.

BECKER H. S. (1970). Sociological Work. Method and Substance. Chicago : Aldine.

BECKER H. S. (2002). Les ficelles du métier. Comment conduire sa recherche en sciences sociales. Paris : La Découverte.

BECKER H. S. (2007). "Préface ». In C. Le Digol (dir.), Dictionnaire de sociologie. Paris : Albin Michel et Encyclopædia Universalis.

BEN AYED C. (2011). "À qui profite le choix de l'école ? Changements d'établissement et destins scolaires des élèves de milieux populaires ». Revue française de pédagogie, no 175, p. 39-58.

BIZEUL D. (1993). Nomades en France. Proximités et clivages. Paris : L'Harmattan.

BLANCHARD M. \& CAYOUETTE-REMBLIÈRE J. (2011). "Penser les choix scolaires ". Revue française de pédagogie, no 175, p. 5-14.

BOUDON R. (1973). L'inégalité des chances. La mobilité sociale dans les sociétés industrielles. Paris: Armand Colin.

BOURDIEU P. (1989). La noblesse d'État. Paris : Éd. de Minuit.

BOURDIEU P. (1997). Méditations pascaliennes. Paris : Éd. du Seuil.
BRIAND J.-P. \& CHAPOULIE J.-M. (2011). Les collèges du peuple. L'enseignement primaire supérieur et le développement de la scolarisation prolongée sous la Troisième République. Rennes: Presses universitaires de Rennes.

BRIAND J.-P., CHAPOULIE J.-M. \& PÉRETZ H. (1980). "L'évolution de la scolarisation post-obligatoire : un schéma d'analyse ». In D. N. Baker \& P. J. Harrigan (dir.), The Making of Frenchmen. Current Directions in the History of Education in France. 1679-1979. Waterloo: Historical Reflections Press, p. 23-53.

BROCCOLICHI S., BEN AYED C. \& TRANCART D. (2006). Les inégalités socio-spatiales d'éducation. Processus ségrégatifs, capital social et politiques territoriales. Rapport à la Direction de la programmation et du développement, ministère de l'Education nationale (dans le cadre de l'appel à projets : "Éducation et formation: disparités territoriales et régionales ").

BROCCOLICHI S., BEN AYED C. \& TRANCART D. (dir.) (2010). École: les pièges de la concurrence. Comprendre le déclin de l'école française. Paris : La Découverte.

BROCCOLICHI S. \& SINTHON R. (2011). "Comment s'articulent les inégalités d'acquisition scolaire et d'orientation ? Relations ignorées et rectifications tardives ». Revue française de pédagogie, no 175, p. 15-38.

CHAPOULIE J.-M. (1987). Les professeurs de l'enseignement secondaire. Un métier de classe moyenne. Paris : Éd. de la Maison des sciences de l'homme.

CORBIN A. (1998). Le monde retrouvé de Louis-François Pinagot. Sur les traces d'un inconnu (1798-1876). Paris : Flammarion.

COUSIN O. (1998). L'efficacité des collèges. Sociologie de l'effet-établissement. Paris : PUF. 
DAVIDSON D. (1993). Actions et événements. Paris : PUF.

DESCOMBES V. (2004). Le complément de sujet. Enquête sur le fait d'agir de soi-même. Paris : Gallimard.

GOMBERT P. \& Van ZANTEN A. (2004). " Le modèle éducatif du pôle "privé" des classes moyennes : ancrages et traductions dans la banlieue parisienne ". Éducation et sociétés, no 14, p. 67-83.

HATZFELD N. (1989). "L'école d'apprentissage Peugeot (1930-1970) : une formation d'excellence ». Formation emploi, no 27-28, p. 115-128.

HÉRIN R. \& ROUAULT R. (1994). Atlas de la France scolaire de la maternelle au lycée. Paris : La Documentation française ; Montpellier : Groupement d'intérêt public Reclus.

HUGHES E. C. (1996). Le regard sociologique. Essais choisis. Paris : Éd. de l'EHESS.

MASSON P. (1999). Les coulisses d'un lycée ordinaire. Enquête sur les établissements secondaires des années 1990. Paris : PUF.

MASSON P. \& SUTEAU M. (2010). «Réinterroger la relation entre école et mobilité sociale. Le cas des enfants d'agriculteurs et d'ouvriers dans l'Ouest ». Sociologie du travail, no 52 , p. 40-54.

OBERTI M. (2007). L'école dans la ville. Ségrégation-mixité-carte scolaire. Paris : Presses de Sciences Po.
PENEFF J. (1987). Écoles publiques, écoles privées dans l'Ouest (1880-1950). Paris : L'Harmattan.

POULLAOUEC T. (2010). « Les habits neufs de l'autoexclusion. Les souhaits d'orientation scolaire des familles ouvrières en fin de 3 e ». Diversité ville école intégration, no 163, p. 1-8.

POULLAOUEC T. (2011). " Choix du destin et destin du choix ». Revue française de pédagogie, no 175, p. 8184.

PROST A. (1992). L'enseignement s'est-il démocratisé ? Paris : PUF.

PROST A. (1993). «Pour une histoire "par en bas" de la scolarisation républicaine ". Histoire de l'éducation, no 57 , p. 59-74.

SAUVY A. \& GIRARD A. (1965). "Les diverses classes sociales devant l'enseignement ». Population, no 20-2, p. 205-232.

SUTEAU M. (1999). Une ville et ses écoles. Nantes, $1830-$ 1940. Rennes: Presses universitaires de Rennes.

QUENSON E. (2001). L'école d'apprentissage Renault. 1919-1989. Paris : CNRS Éd.

Van ZANTEN A. (2009). Choisir son école. Stratégies familiales et médiations locales. Paris : PUF. 